\title{
Proof of Twin Prime Conjecture that can be obtained by using Contradiction method in Mathematics
}

\author{
K.H.K. Geerasee Wijesuriya \\ Research Scientist in Physics and Astronomy
}

PhD student in Astrophysics, Belarusian National Technical University

BSc (Hons) in Physics and Mathematics University of Colombo, Sri Lanka

Doctorate Degree in Physics

geeraseew@gmail.com, geerasee1@gmail.com 


\section{Author's Biography}

The author of this research paper is K.H.K. Geerasee Wijesuriya . And this proof of twin prime conjecture is completely K.H.K. Geerasee Wijesuriya's proof.

Geerasee is 31 years old and Geerasee studied before at Faculty of Science, University of Colombo Sri Lanka. And she graduated with BSc (Hons) in Physics and Mathematics from the University of Colombo, Sri Lanka in 2014. And in March 2018, she completed her first Doctorate Degree in Physics with first class recognition. Now she is following her second $\mathrm{PhD}$ in Astrophysics with Belarusian National Technical University.

Geerasee has been invited by several Astronomy/Physics institutions and organizations worldwide, asking to get involve with them. Also, She has received several invitations from some private researchers around the world asking to contribute to their researches. She worked as Mathematics tutor/Instructor at Mathematics department, Faculty of Engineering, University of Moratuwa, Sri Lanka. Now she is a research scientist in Physics as her career. Furthermore she has achieved several other scientific achievements already.

\section{List of Affiliations}

Faculty of Science, University of Colombo, Sri Lanka, Belarusian National Technical University Belarus

\section{Acknowledgement}

I would be thankful to my parents who gave me the strength to go forward with mathematics and Physics knowledge and achieve my scientific goals.

Keywords: prime; contradiction; greater than ; integer 


\begin{abstract}
Twin prime numbers are two prime numbers which have the difference of 2 exactly. In other words, twin primes is a pair of prime that has a prime gap of two. Sometimes the part "twin prime" is used for a pair of twin primes; an alternative name for this is prime twin or prime pair. Up to date there is no any valid proof/disproof for twin prime conjecture. Through this research paper, my attempt is to provide a valid proof for twin prime conjecture.
\end{abstract}

\title{
Literature Review
}

The question of whether there exist infinitely many twin primes has been one of the great open questions in number theory since many years. This is the content of the twin prime conjecture, which states that there are infinitely many primes $p$ such that $p+2$ number is also prime. In 1849, de Polignac made the more general conjecture that for every natural number $\mathrm{k}$, there are infinitely many primes $\mathrm{p}$ such that $\mathrm{p}+2 \mathrm{k}$ is also prime number. The case $\mathrm{k}=1$ of de Polignac's conjecture is the twin prime conjecture actually.

A stronger form of the twin prime conjecture, the Hardy-Littlewood conjecture, postulates a distribution law for twin primes akin to the prime number theorem indeed. On April 17, 2013, Yitang Zhang (a mathematician) announced a proof that for some integer $\mathrm{N}$ that is less than 70 million, there are infinitely many pairs of primes that differ by his paper was accepted by Annals of Mathematics in early May 2013. Terence Tao who is a removed mathematician, subsequently proposed a Polymath Project collaborative effort to optimize Zhang's bound. As of April 14, 2014, which is one year after Zhang's announcement, the bound has been reduced to 246. Moreover, assuming the Elliott-Halberstam conjecture and its generalized form, the Polymath project wiki states that the bound has been reduced to 12 and 6 , respectively. These improved bounds were discovered using a different approach that was simpler than mathematician Zhang's and was discovered independently by James Maynard and Terence Tao. 


\section{Assumption}

Let's assume that there are finitely many twin prime numbers.

Therefore we proceed by considering that there are finitely many twin prime numbers. Then let the highest twin prime numbers are $\mathrm{P}_{\mathrm{n}-1}$ and $\left(\mathrm{P}_{\mathrm{n}-1}+2\right)$. Then for all prime numbers $\mathrm{P}_{\mathrm{N}}$ greater than $\left(\mathrm{P}_{\mathrm{n}-1}+2\right),\left(\mathrm{P}_{\mathrm{N}}+2\right)$ is not a prime number. Where $\mathrm{N}$ and $\mathrm{n}$ denote two distinct natural numbers. And $\mathrm{P}_{\mathrm{N}}$ denotes the $\mathrm{N}^{\text {th }}$ prime number. $\mathrm{P}_{\mathrm{N}-1}$ denotes $(\mathrm{N}-1)^{\text {th }}$ prime number.

\section{Methodology}

With this mathematical proof, I use the contradiction method to prove that there are infinitely many twin prime numbers.

Let $\mathrm{P}_{\mathrm{n}}$ is an odd number greater than 1 . But let $\mathrm{P}_{3}$ is divisible by $\mathrm{x}_{3}$. But $\mathrm{x}_{3}^{2}$ does not divide $\mathrm{P}_{3}$. And let we choose $P_{n}$ such that $P_{n}=\left[(M+4) / x_{3}\right]-\left(D_{0} \cdot P_{3} / x_{3}\right)$; for some integer $D_{0} \neq 0$. But $\left(D_{0}+2\right)$ is divisible by $x_{3}$. But $\left(D_{0}+2\right)$ is not divisible by $x_{3}^{2}$. But we must have chosen $D_{0}$ integer such that $\mathrm{x}_{3}^{2} \mid\left(\mathrm{P}_{\mathrm{L}} \cdot \mathrm{x}_{3}+\left(\mathrm{D}_{0}+2\right)\right)$ for the non-zero integer $\left.\mathrm{P}_{\mathrm{L}} \ldots \ldots \ldots \ldots \ldots \ldots . . . \ldots . . \ldots\right)$

(To know the meaning of $\mathrm{P}_{\mathrm{L}}$, please refer the below later content)

*** And we have chosen $\mathrm{M}$ such that $\left\{\left[(\mathrm{M}+4) / \mathrm{x}_{3}\right]-\left(\mathrm{D}_{0} \cdot \mathrm{P}_{3} / \mathrm{x}_{3}\right)\right\}\left(=\mathrm{P}_{\mathrm{n}}\right)$ is not divisible by $\mathrm{x}_{3}$ (0)'

*** But we must have chosen $\mathrm{P}_{3}$ and $\mathrm{t}$ such that $\mathrm{P}_{3}=(\mathrm{M}+4) .\left[\left(\mathrm{x}_{3}-1\right) / 2 \mathrm{t}\right] ;$ where $\mathrm{x}^{2}{ }_{3} \mid(\mathrm{t}-1)$ .........(0),

To see the meaning of $\mathrm{P}_{3}, \mathrm{x}_{3}, \mathrm{t}$ and $\mathrm{M}$, please refer the below content.

Let's show that the conditions in (0)' and in (0)' 'both are possible simultaneously in the proof as below:

Let we choose odd integer ' $a$ ' such that ' $a$ ' is not divisible by $\mathrm{x}_{3}$. Let choose integer $\mathrm{P}_{\mathrm{Q}}$ such that $\mathrm{P}_{\mathrm{Q}}=\mathrm{a}+\mathrm{a} \cdot \mathrm{x}_{3}{ }^{2}$ (Then $\mathrm{P}_{\mathrm{Q}}$ is not divisible by $\mathrm{x}_{3}$ since ' $\mathrm{a}$ ' is not divisible by $\mathrm{x}_{3}$ ). Then $\mathrm{P}_{\mathrm{Q}}$ is an even number. i.e. $(M+4)$ is an even integer. 
Where we are responsible to choose $\mathrm{P}_{\mathrm{Q}}$ such that $\left[\mathrm{P}_{\mathrm{Q}}-\mathrm{D}_{0} \cdot\left(\mathrm{P}_{3} / \mathrm{x}_{3}\right)\right]$ is not divisible by $\mathrm{x}_{3}$.

Where $P_{Q}=(M+4) / x_{3}$.

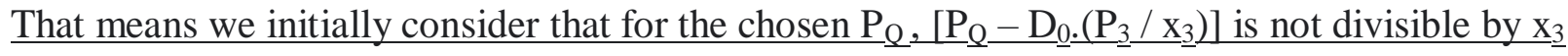

Then $P_{Q} /(2 . a)=(M+4) /\left(2 \cdot a \cdot x_{3}\right)=$ non-zero integer, since $(M+4)$ is an even number. Let choose integer $t(\neq 0)$ such that $t=\left(P_{Q} / a\right)$.

Then $[(\mathrm{M}+4) / 2 . \mathrm{t}]=\mathrm{P}_{\mathrm{Q}} \cdot \mathrm{x}_{3} / 2 \mathrm{t}=\mathrm{P}_{\mathrm{Q}} \cdot \mathrm{x}_{3} \cdot \mathrm{a} /\left(2 \cdot \mathrm{P}_{\mathrm{Q}}\right)=\mathrm{a} \cdot \mathrm{x}_{3} / 2 \ldots \ldots \ldots \ldots(0), "$,

We have chosen integer $P_{3}(\neq 0)$ such that $P_{3}=(M+4) \cdot\left[\left(x_{3}-1\right) / 2 \cdot t\right]=\left(x_{3}-1\right) \cdot a \cdot x_{3} / 2$ (= integer, since $\left(\mathrm{x}_{3}-1\right)$ is an even number). Then $\mathrm{P}_{3}$ is divisible by $\mathrm{x}_{3}$. But $\mathrm{P}_{3}$ is not divisible by $\mathrm{x}^{2}$.

Then t.a. $\mathrm{x}_{3}=(\mathrm{M}+4)\left(\right.$ by $\left.(0)^{\prime \prime \prime}\right)$.

And then $\mathrm{P}_{3}=(\mathrm{a} / 2) \cdot \mathrm{x}_{3} \cdot\left(\mathrm{x}_{3}-1\right)$; here ' $\mathrm{a}$ ' is an odd integer $(\neq 0)$ which is not divisible by $\mathrm{x}_{3}$.

But $2 \mid\left(\mathrm{x}_{3}-1\right)$ and 4 does not divide $\left(\mathrm{x}_{3}-1\right)$. ***See the Proof 1 below to see the existence of $\left(\mathrm{P}_{\mathrm{N}}-2\right)=\mathrm{P}_{3} \cdot \mathrm{x}_{3}$ such that $\mathrm{P}_{3}=(\mathrm{a} / 2) \cdot \mathrm{x}_{3} \cdot\left(\mathrm{x}_{3}-1\right)$ for some odd integer a ; where 4 does not divide $\left(\mathrm{x}_{3}-1\right)$.

Thus still $\mathrm{P}_{3}\left(=(\mathrm{a} / 2) \cdot \mathrm{x}_{3} \cdot\left(\mathrm{x}_{3}-1\right)\right)$ is an odd number.

Then $(\mathrm{t}-1)=\left(\mathrm{P}_{\mathrm{Q}} / \mathrm{a}\right)-1=\left[\left(\mathrm{P}_{\mathrm{Q}}-\mathrm{a}\right) / \mathrm{a}\right]=\mathrm{x}_{3}{ }^{2}$. Then $(\mathrm{t}-1)=\mathrm{x}_{3}{ }^{2}$. Then $(\mathrm{t}-1)$ is divisible by $\mathbf{x}_{3}^{2}$. But $\mathrm{P}_{\mathrm{n}}=\mathrm{P}_{\mathrm{Q}}-\mathrm{D}_{0} \cdot\left(\mathrm{P}_{3} / \mathrm{x}_{3}\right) \ldots(0.0 .1)$

But since ' $a$ ' is not divisible by $\mathrm{x}_{3}$, [a. $\left.\left(\mathrm{x}_{3}-1\right) / 2\right]$ is not divisible by $\mathrm{x}_{3}$.

And we chose $\mathrm{P}_{\mathrm{Q}}\left(=\mathrm{a}+\mathrm{a} \cdot \mathrm{x}_{3}{ }_{3}\right)$ such that $\mathrm{P}_{\mathrm{Q}}-\mathrm{D}_{0} \cdot\left(\mathrm{P}_{3} / \mathrm{x}_{3}\right)$ is not divisible by $\mathrm{x}_{3}$. $\mathrm{By}(0.0 .1)$ : the given $P_{n}$ in $(0.0 .1)$ is still not divisible by $x_{3}$.

That means we choose $P_{Q}\left(=a+a . x^{2}\right)$ and $\left[P_{Q}-D_{0} \cdot\left(P_{3} / x_{3}\right)\right]$ is not divisible by $x_{3} \rightarrow$ possible to have the integer $t$ such that $(t-1)=x_{3}{ }^{2}$ whenever $t=(M+4)$. $\left[\left(x_{3}-1\right) / 2 . P_{3}\right]$

Thus the conditions in (0)' and in (0)', both are possible simultaneously in the proof. \} 
Let $\mathrm{P}_{\mathrm{N}}$ is an arbitrary prime number greater than $\left(\mathrm{P}_{\mathrm{n}-1}+2\right)$. Because there are infinitely many prime numbers. And here $\left(\mathrm{P}_{\mathrm{N}}-2\right)>\left(\mathrm{P}_{\mathrm{n}-1}+2\right)$. Thus $\left(\mathrm{P}_{\mathrm{N}}-2\right)$ is not a prime number.

Where each arbitrary $P_{N}$ prime number obey (that means we choose a set of $P_{N}\left(>\left(P_{n-1}+2\right)\right)$ arbitrarily such that each arbitrarily chosen $\mathrm{P}_{\mathrm{N}}$ give us :

" $\mathrm{P}_{3}$ is divisible by $\mathrm{x}_{3}$. But $\mathrm{x}^{2}{ }_{3}$ does not divide $\mathrm{P}_{3}$; whenever $\left(\mathrm{P}_{\mathrm{N}}-2\right)=\mathrm{P}_{3} \cdot \mathrm{x}_{3}$ "

And here $\left(\mathrm{P}_{\mathrm{N}}+2\right)>\left(\mathrm{P}_{\mathrm{n}-1}+2\right)$. Then according to our assumption, $\left(\mathrm{P}_{\mathrm{N}}+2\right)$ is also not a prime number. Here $\mathrm{P}_{N}$ is a prime number such that $\left(\mathrm{P}_{\mathrm{N}}+2\right)$ is dividing by prime number $\mathrm{P}_{2}$.

Thus $\left(\mathrm{P}_{\mathrm{N}}+2\right)=\mathrm{P}_{2} * \mathrm{x}_{2}$ for some $\mathrm{x}_{2}$ natural number. Since $\mathrm{P}_{\mathrm{N}}$ is a prime number, for some $\mathrm{r}_{2}$ (rational number which is not a natural number): $\mathrm{P}_{\mathrm{N}} / \mathrm{r}_{2}=\mathrm{P}_{2}$. Thus $\left(\mathrm{P}_{\mathrm{N}}+2\right)=\mathrm{P}_{2} * \mathrm{x}_{2}$ (02) and $\mathrm{P}_{\mathrm{N}}=\mathrm{r}_{2} * \mathrm{P}_{2}$

$\mathrm{X}_{2}$ is a natural number and $\mathrm{P}_{2}$ is a prime number. Since $\mathrm{P}_{\mathrm{N}}$ is a prime number, $\left(\mathrm{P}_{\mathrm{N}}-2\right)$ is also not a prime number ( Since $P_{N}-2>P_{n-1}+2$ ). Then for some integer $P_{3}$ greater than 1 such that $\left(P_{N}-2\right) / P_{3}=x_{3} ;$ where $x_{3}$ is an integer greater than 1 . But here we considered that $x_{3} \mid P_{3}$.

$\left(\mathrm{P}_{\mathrm{N}}-2\right)=\mathrm{P}_{3} * \mathrm{x}_{3}$

But $\left(\mathrm{P}_{\mathrm{N}}+2\right), \mathrm{P}_{\mathrm{n}}$ both are odd numbers. Thus $\left(\mathrm{P}_{\mathrm{N}}+2\right)=\mathrm{P}_{\mathrm{n}}+2 . l$; for some $l$ integer $($ where $l \neq 0)$ (05)

Then $\left(\mathrm{P}_{\mathrm{N}}-2\right)=\mathrm{P}_{\mathrm{n}}+2 . l-4=\mathrm{P}_{\mathrm{n}}+2 .(l-2)$

And we know that $\left(\mathrm{P}_{\mathrm{N}}+2\right)=\mathrm{P}_{\mathrm{n}}+2 . l \rightarrow \mathrm{P}_{\mathrm{N}}=\mathrm{P}_{\mathrm{n}}+2 . l-2$

Thus by (*): $\mathrm{P}_{\mathrm{n}}+2 . l-2=\mathrm{P}_{\mathrm{N}}$. Thus by $(04)$ and $(*): \mathrm{P}_{3} * \mathrm{x}_{3}+2=\mathrm{P}_{\mathrm{n}}+2 . l-2$

Thus $\mathrm{P}_{3} * \mathrm{x}_{3}-2 . l+4=\mathrm{P}_{\mathrm{n}}$

Thus $\mathrm{P}_{3} * \mathrm{x}_{3}+2 .(l-2)=\mathrm{P}_{\mathrm{n}}+4 .(l-2)=\mathrm{P}_{\mathrm{n}}+2 \cdot \mathrm{P}_{\mathrm{N}}-4-2 \cdot \mathrm{P}_{\mathrm{n}}=2 \cdot \mathrm{P}_{\mathrm{N}}-4-\mathrm{P}_{\mathrm{n}}\left(\right.$ by $\left.(6.1)^{\prime}\right)$

Thus $\mathrm{P}_{3} * \mathrm{x}_{3}+2 \cdot(l-2)=2 \cdot \mathrm{P}_{\mathrm{N}}-4-\mathrm{P}_{\mathrm{n}}=\mathrm{P}_{\mathrm{n}}$,

Thus $\mathrm{P}_{3} * \mathrm{x}_{3}+2 .(l-2)=\mathrm{P}_{\mathrm{n}},=2 . \mathrm{P}_{3} * \mathrm{x}_{3}-\mathrm{P}_{\mathrm{n}}$

Thus $\mathrm{P}_{3} * \mathrm{x}_{3}+2 . l=4+2 . \mathrm{P}_{3} * \mathrm{x}_{3}-\mathrm{P}_{\mathrm{n}}$ 
$\mathrm{P}_{3} * \mathrm{x}_{3}+(2 . l+\mathrm{M})=\left(4+\mathrm{M}-\mathrm{P}_{\mathrm{n}}\right)+2 . \mathrm{P}_{3} * \mathrm{x}_{3} ;$ Where $\mathrm{M}$ is an integer $(\mathrm{M} \neq 0)$

$(2 . l+M)=\left(4+M-P_{n}\right)+P_{3} * x_{3} ;$ Where $M$ is an integer $\neq 0$

But we chose $M$ such that $(M+4)$ is divisible by $x_{3}$. But $(M+4)$ is not divisible by $x^{2}$.

But we know that $\mathrm{P}_{3}$ is divisible by $\mathrm{x}_{3}$. But $\mathrm{x}_{3}^{2}$ does not divide $\mathrm{P}_{3}$. And we know that $\left(\mathrm{P}_{3} * \mathrm{x}_{3}\right)$ is divisible by $\mathrm{x}_{3}$. And we know that $\mathrm{P}_{\mathrm{n}}$ is not divisible by $\mathrm{x}_{3}$.......

Thus by (8): $\mathrm{x}_{3}$ does not divide $\left(2 . l+\mathrm{M}\right.$ ). Since $\mathrm{P}_{3}$ is divisible by $\mathrm{x}_{3}, \mathrm{P}_{3}$ does not divide $(2 . l+\mathrm{M})$

But $\mathrm{P}_{\mathrm{N}}$ is an arbitrary prime greater than $\left(\mathrm{P}_{\mathrm{n}-1}+2\right)$. Then let $\left\{\left(\mathrm{P}_{\underline{N}}+\mathrm{A}_{1}\right), \mathrm{P}_{\mathrm{N}}\right\}$ are two arbitrary consecutive primes set such that each primes are greater than $\left(\mathrm{P}_{\underline{n-1}}+2\right)$. Where each arbitrary $\mathrm{P}_{\underline{N}}$ prime number obey:

" $\mathrm{P}_{3}$ is divisible by $\mathrm{x}_{3}$. But $\mathrm{x}_{3}^{2}$ does not divide $\mathrm{P}_{3}$; whenever $\left(\mathrm{P}_{\mathrm{N}}-2\right)=\mathrm{P}_{3} \cdot \mathrm{x}_{3}$ "

Here since $\mathrm{P}_{N}>\left(\mathrm{P}_{\mathrm{n}-1}+2\right)$ and since $\left(\mathrm{P}_{\mathrm{N}}+\mathrm{A}_{1}\right)>\left(\mathrm{P}_{\mathrm{n}-1}+2\right), \mathrm{A}_{1} \neq(+/-) 2$. Because for any two arbitrary consecutive primes greater than $\left(\mathrm{P}_{\mathrm{n}-1}+2\right)$, the difference between those consecutive primes is greater than 2 ( ince the greatest twin primes are $\mathrm{P}_{\mathrm{n}-1}$ and $\left[\mathrm{P}_{\mathrm{n}-1}+2\right]$ ).

But $A_{1} \neq 2$. $\left(\mathrm{x}_{3}-1\right)$. But now choose two particular two consecutive primes (greater than $\left.\left(\mathrm{P}_{\mathrm{n}-1}+2\right)\right)$ from the arbitrary prime number set $\left\{\left(\mathrm{P}_{\mathrm{N}}+\mathrm{A}_{1}\right), \mathrm{P}_{\mathrm{N}}\right\}$ such that those chosen two particular consecutive primes obey $\left[\mathrm{P}_{3} \mid\left(\mathrm{A}_{1}-2\right)\right]$. i.e. where particularly we choose $\mathrm{A}_{1}$ such that $\mathrm{P}_{\mathrm{L}}-\mathrm{x}_{3}=$ $B_{2}$. Where $\left(A_{1}-2\right) / P_{3}=B_{2}$. Where $P_{L}=(P-4) / P_{3}$. Here $P=$ chosen particular prime $\left(P_{N}+A_{1}\right)$. Since $A_{1} \neq-2$, there exists an odd number $P_{3}$ greater than 1 such that $\left[P_{3} \mid\left(A_{1}-2\right)\right]$. Refer the 'Proof' below to see the existence of two consecutive primes $\left(\mathbf{P}_{N}+A_{1}\right)$ and $\mathbf{P}_{N}$ such that [ $\left.P_{3} \mid\left(A_{1}-2\right)\right]$. And refer 'Proof 1 ' to see the existence of an integer $\left(P_{N}-2\right)$ such that $\left(P_{N}-2\right)=P_{3} \cdot x_{3}$ such that $P_{3}$ is divisible by $x_{3}$. But $x_{3}{ }_{3}$ does not divide $P_{3}$.

But we know that $\left(P_{N}+A_{1}\right)>\left(P_{n-1}+2\right)$. Thus here $A_{1} \neq(+/-) 2$, since there are finite number of twin primes according to our assumption. BUT REMEMBER THAT $\mathrm{P}_{\mathrm{N}}$ AND $\left(\mathrm{P}_{\mathrm{N}}+\mathrm{A}_{1}\right)$ ARE CONSECUTIVE PRIMES greater than $\left(\mathrm{P}_{\mathrm{n}-1}+2\right)$.

$\left\{\right.$ Here $\left(\mathrm{P}_{\mathrm{N}}-2\right)=\mathrm{P}_{3 .} \mathrm{X}_{3}$ and $\left(\mathrm{P}_{\mathrm{N}}+\mathrm{A}_{1}\right)=\mathrm{P}=$ Prime. That means $\mathrm{P}_{3 .} \mathrm{X}_{3}+\left(\mathrm{A}_{1}+2\right)=\mathrm{P}$

But $\left(A_{1}-2\right)$ is divisible by $P_{3}$. Thus $\left(A_{1}+2\right)$ is not divisible by $P_{3}$. Because $P_{3}$ does not divide 4 . 
But since $\mathrm{P}_{3} * \mathrm{x}_{3}$ is divisible by $\mathrm{P}_{3}, \mathrm{P}$ is not divisible by $\mathrm{P}_{3}$.

But $\left(A_{1}-2\right)$ is divisible by $P_{3}$ and since $\left(x_{3} \mid P_{3}\right), x_{3} \mid\left(A_{1}-2\right)$. Thus $\left(A_{1}+2\right)$ is not divisible by $\mathrm{x}_{3}$. Because $\mathrm{x}_{3}$ does not divide 4 since $\mathrm{x}_{3}$ is an odd number $\left(\right.$ since $\left.\left(\mathrm{P}_{\mathrm{N}}-2\right)=\mathrm{P}_{3} \cdot \mathrm{x}_{3}\right)$.

But since $\mathrm{P}_{3} * \mathrm{x}_{3}$ is divisible by $\mathrm{x}_{3}, \mathrm{P}$ is not divisible by $\mathrm{x}_{3}$.

But $\mathrm{P}=\mathrm{P}_{3} \mathrm{X}_{3}+\mathrm{A}_{1}+2 \neq \mathrm{P}_{3} \mathrm{x}_{3}+2 \cdot\left(\mathrm{x}_{3}-1\right)+2=\mathrm{P}_{3} \mathrm{x}_{3}+2 \cdot \mathrm{x}_{3}=\mathrm{x}_{3} \cdot\left(\mathrm{P}_{3}+2\right)$. Thus $\mathrm{P} \neq \mathrm{x}_{3} \cdot\left(\mathrm{P}_{3}+2\right)$.

Therefore according to above steps, we can write $\mathrm{P}_{3 .} \mathrm{X}_{3}+\left(\mathrm{A}_{1}+2\right)=\mathrm{P}$ as a prime $\left.\quad\right\}$

But $(2 . l+M)=P_{N}-P_{n}+2+M=\left(P_{N}+A_{1}\right)+\left(M+2-A_{1}-P_{n}\right) \ldots$

By (8.1): $\mathrm{x}_{3} \mid(\mathrm{M}+4)$. But $\left[\mathrm{P}_{3} \mid\left(\mathrm{A}_{1}-2\right)\right]$.

But since $\left[\mathrm{P}_{3} \mid\left(\mathrm{P}_{\mathrm{N}}-2\right)\right]$ and since $\mathrm{P}_{3}$ does not divide $\left(\mathrm{A}_{1}+2\right),\left\{\left(\mathrm{A}_{1}+2\right)+\left(\mathrm{P}_{\mathrm{N}}-2\right)\right\}$ does not divide by $\mathrm{P}_{3}$. i.e. $\mathrm{P}\left(=\left(\mathrm{P}_{\mathrm{N}}+\mathrm{A}_{1}\right)\right)$ does not divide by $\mathrm{P}_{3}$. Thus our choice of $\mathrm{A}_{1}$ such that $\left[\mathrm{P}_{3} \mid\left(\mathrm{A}_{1}-2\right)\right]$ is okay.

But $\left[\mathrm{P}_{3} \mid\left(\mathrm{P}_{\mathrm{N}}-2\right)\right]$ and $\left[\mathrm{P}_{3} \mid\left(\mathrm{A}_{1}-2\right)\right]$. Thus $\left(\mathrm{P}_{\mathrm{N}}-2\right)=\mathrm{P}_{3} \cdot \mathrm{x}_{3}$ and $\left(\mathrm{A}_{1}-2\right)=\mathrm{P}_{3} \cdot \mathrm{B}_{2}$; where $\mathrm{x}_{3}$ and $\mathrm{B}_{2}$ are integers and each of them not equals to 0 .

Thus $\left(\mathrm{P}_{\mathrm{N}}+\mathrm{A}_{1}-4\right)=\mathrm{P}_{3} \cdot \mathrm{X}_{3}+\mathrm{P}_{3} \cdot \mathrm{B}_{2}=(\mathrm{P}-4)$

i.e $\mathrm{P}_{3} \mid(\mathrm{P}-4)$.

Let's consider $M$ integer such that $M=P-C$; for some integer ' $C$ ' $\neq 0$

But $\mathrm{x}_{3} \mid(\mathrm{M}+4)$ and $\mathrm{P}_{3} \mid(\mathrm{P}-4)$ by (8.1) and (11).

By (12): $\mathrm{P}=(\mathrm{M}+\mathrm{C})$. Thus $[(\mathrm{M}+\mathrm{C})]-4=\mathrm{P}_{3} . \mathrm{P}_{\mathrm{L}}$

Where $\mathrm{P}_{\mathrm{L}}=\left[(\mathrm{P}-4) / \mathrm{P}_{3}\right]=$ integer, but not equals to 0 .

Then $\left(\mathrm{P}_{\mathrm{L}} / \mathrm{x}_{3}\right)=\left[(\mathrm{P}-4) /\left(\mathrm{x}_{3} \mathrm{P}_{3}\right)\right]=(\mathrm{P}-4) /\left(\mathrm{P}_{\mathrm{N}}-2\right)$

$=\left[\left(\mathrm{P}_{\mathrm{N}}-2\right)+\left(\mathrm{A}_{1}-2\right)\right] /\left(\mathrm{P}_{\mathrm{N}}-2\right)=1+\left[\left(\mathrm{A}_{1}-2\right) /\left(\mathrm{P}_{\mathrm{N}}-2\right)\right]$

If $A_{1}<0$, then either $1+\left[\left(A_{1}-2\right) /\left(P_{N}-2\right)\right]<0$ or $0<1+\left[\left(A_{1}-2\right) /\left(P_{N}-2\right)\right]<1$. 
But $\mathrm{P}_{\mathrm{L}}=\left[(\mathrm{P}-4) / \mathrm{P}_{3}\right]$. Since $(\mathrm{P}-4)>0$ and since $\mathrm{x}_{3}>1, \mathrm{P}_{3}>1 ; \mathrm{P}_{\mathrm{L}}>0$. Thus it is impossible to have $1+\left[\left(\mathrm{A}_{1}-2\right) /\left(\mathrm{P}_{\mathrm{N}}-2\right)\right]<0$. Thus if $\mathrm{A}_{1}<0$, we have $0<1+\left[\left(\mathrm{A}_{1}-2\right) /\left(\mathrm{P}_{\mathrm{N}}-2\right)\right]<1$. Then $\left(P_{L} / x_{3}\right)$ is not an integer. If $A_{1}>0$, then $A_{1}>2$. Then $\left(P_{N}-A_{1}\right)<\left(P_{N}-2\right)$.

Then $\left(\mathrm{P}_{\mathrm{L}} / \mathrm{x}_{3}\right)=1+\left[\left(\mathrm{A}_{1}-2\right) /\left(\mathrm{P}_{\mathrm{N}}-2\right)\right]=1+\left[\left(\mathrm{A}_{1}-\mathrm{P}_{\mathrm{N}}+\mathrm{P}_{\mathrm{N}}-2\right) /\left(\mathrm{P}_{\mathrm{N}}-2\right)\right]$

$=2+\left[\left(\mathrm{A}_{1}-\mathrm{P}_{\mathrm{N}}\right) /\left(\mathrm{P}_{\mathrm{N}}-2\right)\right]=2-\left[\left(\mathrm{P}_{\mathrm{N}}-\mathrm{A}_{1}\right) /\left(\mathrm{P}_{\mathrm{N}}-2\right)\right]$.

Since $A_{1} \neq(+/-) 2$ and by (13)' : $\left[\left(P_{N}-A_{1}\right) /\left(P_{N}-2\right)\right]$ is not an integer.

Then $2-\left[\left(\mathrm{P}_{\mathrm{N}}-\mathrm{A}_{1}\right) /\left(\mathrm{P}_{\mathrm{N}}-2\right)\right]=\left(\mathrm{P}_{\mathrm{L}} / \mathrm{x}_{3}\right)$ is not an integer.

Then for $A_{1}>0$ and for $A_{1}<0:\left(P_{L} / x_{3}\right)$ is not an integer.

Thus here $\mathrm{x}_{3}$ does not divide $\mathrm{P}_{\mathrm{L}}$

But $\left[(\mathrm{M}+4) / \mathrm{x}_{3}\right]=\mathrm{P}_{\mathrm{Q}}=$ integer, but not equals to 0 .

Thus by (13): $\left[\left(\mathrm{x}_{3} \cdot \mathrm{P}_{\mathrm{Q}}-4+\mathrm{C}\right)\right]-4=\mathrm{P}_{3} \cdot \mathrm{P}_{\mathrm{L}}$

Thus $\mathrm{C}-4=\left[\left(\mathrm{P}_{3} \cdot \mathrm{P}_{\mathrm{L}}+4\right)-\mathrm{x}_{3} \cdot \mathrm{P}_{\mathrm{Q}}\right]$

By (09): $(2 . l+M)=\left(P_{N}+A_{1}\right)+\left(M+2-A_{1}-P_{n}\right)=P+\left(M+2-A_{1}-P_{n}\right)$

$=\mathrm{P}+\mathrm{P}-\mathrm{C}+2-\mathrm{A}_{1}-\mathrm{P}_{\mathrm{n}}=2 \cdot \mathrm{P}-\mathrm{C}-\mathrm{P}_{3} \cdot \mathrm{B}_{2}-\mathrm{P}_{\mathrm{n}}$

By (14): $C=\left[\left(P_{3} \cdot P_{L}+8\right)-x_{3} \cdot P_{Q}\right]$. Then 2.P $-C-P_{n}=2 \cdot P+x_{3} \cdot P_{Q}-\left(P_{3} \cdot P_{L}+8\right)-P_{n}$

$=2 \cdot(P-4)+x_{3} \cdot P_{Q}-P_{n}-\left(P_{3} \cdot P_{L}\right)=2 \cdot P_{3} \cdot P_{L}+x_{3} \cdot P_{Q}-P_{n}-\left(P_{3} \cdot P_{L}\right)=P_{3} \cdot P_{L}+\left[x_{3} \cdot P_{Q}-P_{n}\right]$

$=\mathrm{x}_{3} \cdot \mathrm{P}_{3} \cdot\left\{\left(\mathrm{P}_{\mathrm{L}} / \mathrm{x}_{3}\right)+\left[\left(\mathrm{P}_{\mathrm{Q}} / \mathrm{P}_{3}\right)-\left(\mathrm{P}_{\mathrm{n}} /\left(\mathrm{x}_{3} \cdot \mathrm{P}_{3}\right)\right)\right]\right\}$

But we chose $P_{n}$ such that $\left(x_{3} \cdot P_{n}\right)=(M+4)-D_{0} \cdot P_{3}$; for some integer $D_{0}$.

$* * *$ But we have chosen $\mathrm{D}_{0}$ integer such that $\mathrm{x}_{3}{ }_{3} \mid\left(\mathrm{P}_{\mathrm{L}} \cdot \mathrm{x}_{3}+\left(\mathrm{D}_{0}+2\right)\right)$. Where $\mathrm{D}_{0} \neq 0$ and $\left(\mathrm{D}_{0}+2\right)$ is divisible by $x_{3}$ (it implies that $\mathrm{D}_{0}$ is not divisible by $\mathrm{x}_{3}$ ). But $\left(\mathrm{D}_{0}+2\right)$ is not divisible by $\mathrm{x}_{3}^{2}$.

And here $\left(\mathrm{P}_{\mathrm{L}} / \mathrm{x}_{3}\right)+\left[\left(\mathrm{D}_{0}+2\right) / \mathrm{x}_{3}^{2}\right]=\mathrm{D}^{\prime}$ for some integer $\mathrm{D}^{\prime}$.

Since $\mathrm{P}_{\mathrm{L}}$ is not divisible by $\mathrm{x}_{3}$, there exists such an integer number $\left(\mathrm{D}_{0}+2\right)$ (not equals to zero), such that $\mathrm{x}_{3}{ }_{3} \mid\left(\mathrm{P}_{\mathrm{L}} \cdot \mathrm{x}_{3}+\left(\mathrm{D}_{0}+2\right)\right)$; whenever $\left(2+\mathrm{D}_{0}\right)$ is not divisible by $\mathrm{x}_{3}{ }_{3}$.

Then $\mathrm{P}_{\mathrm{n}}=\left[(\mathrm{M}+4) / \mathrm{x}_{3}\right]-\left(\mathrm{D}_{0} \cdot \mathrm{P}_{3} / \mathrm{x}_{3}\right)$. Then $\left[\left(\mathrm{P}_{\mathrm{Q}} / \mathrm{P}_{3}\right)-\left(\mathrm{P}_{\mathrm{n}} / \mathrm{P}_{3}\right)\right]=\left(\mathrm{D}_{0} / \mathrm{x}_{3}\right)$ 
$=\left[\mathrm{x}_{3} .\left(\mathrm{P}_{\mathrm{Q}} / \mathrm{P}_{3}\right)-\left(\mathrm{P}_{\mathrm{n}} / \mathrm{P}_{3}\right)\right]-\left(\mathrm{x}_{3}-1\right) .\left(\mathrm{P}_{\mathrm{Q}} / \mathrm{P}_{3}\right)$

Then $\left[\mathrm{x}_{3} .\left(\mathrm{P}_{\mathrm{Q}} / \mathrm{P}_{3}\right)-\left(\mathrm{P}_{\mathrm{n}} / \mathrm{P}_{3}\right)\right]=\left(\mathrm{D}_{0} / \mathrm{x}_{3}\right)+\left(\mathrm{x}_{3}-1\right) .\left(\mathrm{P}_{\mathrm{Q}} / \mathrm{P}_{3}\right)$

Then $\left[\left(\mathrm{P}_{\mathrm{Q}} / \mathrm{P}_{3}\right)-\left(\mathrm{P}_{\mathrm{n}} /\left(\mathrm{x}_{3} \cdot \mathrm{P}_{3}\right)\right)\right]=\left(\mathrm{D}_{0} / \mathrm{x}_{3}^{2}\right)+\left(\mathrm{x}_{3}-1\right) \cdot\left(\mathrm{P}_{\mathrm{Q}} /\left(\mathrm{x}_{3} \cdot \mathrm{P}_{3}\right)\right)=$

$\left(\mathrm{D}_{0} / \mathrm{x}_{3}^{2}\right)+\left(\mathrm{x}_{3}-1\right) \cdot\left(\mathrm{P}_{\mathrm{Q}} /\left(\mathrm{x}_{3}^{2} \cdot \mathrm{u}\right)\right)=\left[\mathrm{D}_{0} \cdot \mathrm{u}+\left(\mathrm{x}_{3}-1\right) \cdot \mathrm{P}_{\mathrm{Q}}\right] /\left(\mathrm{u} \cdot \mathrm{x}_{3}^{2}\right) \ldots \ldots .(16 \cdot 0)$

where $u . x_{3}=P_{3}$, then $u$ is not divisible by $x_{3}$.

But we chose (t. $\left.P_{3}\right)=(M+4)$. $\left[\left(x_{3}-1\right) / 2\right] ; t$ is an integer $\neq 0$ such that $x^{2}{ }_{3} \mid(t-1)$.

Then $\left.\left(\mathrm{P}_{3} / \mathrm{x}_{3}\right)=\mathrm{u}=(\mathrm{M}+4) .\left(\mathrm{x}_{3}-1\right)\right] /\left(2 \cdot \mathrm{t} \cdot \mathrm{x}_{3}\right)=$ integer

Then $\left.\mathrm{u}=\mathrm{P}_{\mathrm{Q}} \cdot\left(\mathrm{x}_{3}-1\right)\right] / 2 . \mathrm{t}$

Thus by (16.0): $\left[\left(\mathrm{P}_{\mathrm{Q}} / \mathrm{P}_{3}\right)-\left(\mathrm{P}_{\mathrm{n}} /\left(\mathrm{x}_{3} . \mathrm{P}_{3}\right)\right)\right]=\left[\mathrm{D}_{0} \cdot \mathrm{u}+2 . \mathrm{t} . \mathrm{u}\right] /\left(\mathrm{u} . \mathrm{x}_{3}{ }_{3}\right)=\left(\mathrm{D}_{0}+2 \mathrm{t}\right) / \mathrm{x}^{2}{ }_{3}$; where $\mathrm{P}_{\mathrm{Q}}$ is not divisible by $\mathrm{x}_{3}$. Then according to the formula for $\mathrm{u}$, still ' $\mathrm{u}$ ' is not divisible by $\mathrm{x}_{3}$.

Then $\left\{\left(\mathrm{P}_{\mathrm{L}} / \mathrm{x}_{3}\right)+\left[\left(\mathrm{P}_{\mathrm{Q}} / \mathrm{P}_{3}\right)-\left(\mathrm{P}_{\mathrm{n}} /\left(\mathrm{x}_{3} \cdot \mathrm{P}_{3}\right)\right)\right]\right\}=\left(\mathrm{P}_{\mathrm{L}} / \mathrm{x}_{3}\right)+\left[\left(\mathrm{D}_{0}+2 \mathrm{t}\right) / \mathrm{x}_{3}^{2}\right]$

$=\left(\mathrm{P}_{\mathrm{L}} / \mathrm{x}_{3}\right)+\left[\left(\mathrm{D}_{0}+2\right) / \mathrm{x}_{3}^{2}\right]+\left[2 .(\mathrm{t}-1) / \mathrm{x}_{3}^{2}\right]=\left[\left[\mathrm{P}_{\mathrm{L}} \cdot \mathrm{x}_{3}+\left(\mathrm{D}_{0}+2\right)\right] / \mathrm{x}_{3}^{2}\right]+\mathrm{t}^{\prime}=\mathrm{D}^{\prime}=$ integer number $\left(\right.$ since $\mathrm{t}^{\prime}=2 .(\mathrm{t}-1) / \mathrm{x}_{3}^{2}=$ integer $)$.

Since $\mathrm{P}_{\mathrm{L}}$ is not divisible by $\mathrm{x}_{3}$, there exists such an integer number $\mathrm{D}_{0}$ (not equals to zero), such that $\mathrm{x}_{3}{ }_{3} \mid\left(\mathrm{P}_{\mathrm{L} \cdot \mathrm{x}_{3}}+\left(\mathrm{D}_{0}+2\right)\right)$. Whenever $\left(2+\mathrm{D}_{0}\right)$ is divisible by $\mathrm{x}_{3}$. Whenever $\left(2+\mathrm{D}_{0}\right)$ is not divisible by $\mathrm{x}_{3}{ }_{3}$.

Then by (16): 2.P $-C-P_{n}=P_{3} . x_{3}\left[D^{\prime}\right]$; where $\left(P_{L} / x_{3}\right)$ and $\left(D_{0}+2\right) / x^{2}$ are not integers (by 13.1). But we chose $D_{0}$ such that $x_{3}^{2} \mid\left(P_{L} \cdot x_{3}+\left(D_{0}+2\right)\right)$. Then 2.P $-C-P_{n}=P_{3} \cdot x_{3}$. D' ;

where $D^{\prime}=\left(P_{L} \cdot x_{3}+\left(D_{0}+2\right)\right) / x_{3}^{2}+t^{\prime}=$ integer, but not equals to 0 .

Then $\mathrm{P}_{3} \mid\left(2 . \mathrm{P}-\mathrm{C}-\mathrm{P}_{\mathrm{n}}\right)$

by (15), (17): $\mathrm{P}_{3} \mid(2 . l+\mathrm{M})$

Thus by (i): $\mathrm{P}_{3}$ does not divide $(2 . l+\mathrm{M})$. ..(19). Thus by (18) and (19): We have a contradiction

Therefore the only possibility is: our assumption (1.0) is false. Therefore there are infinitely many Twin Prime Numbers. 


\section{Proof}

Let's prove that there exists consecutive primes $P_{N}$ and $\left(P_{N}+A_{1}\right)$ such that $\left[P_{3} \mid\left(A_{1}-2\right)\right]$ for some odd integer $\mathrm{P}_{3}$ which is greater than 1 (when there exist consecutive prime numbers $\mathrm{P}_{\mathrm{N}}$ and $\left(\mathrm{P}_{\mathrm{N}}+\mathrm{A}_{1}\right)$ which both are greater than $\left.\left[\mathrm{P}_{\mathrm{n}-1}+2\right]\right)$ through this 'Proof' as below.

By $2^{\text {nd }}$ reference: $\mathrm{P}_{\mathrm{N}-1}=\left(\mathrm{P}_{\mathrm{N}}+\mathrm{A}_{1}\right)=2+\sum_{j=1}^{N-2} h j$, where $\mathrm{h}_{\mathrm{j}}=\mathrm{P}_{\mathrm{j}+1}-\mathrm{P}_{\mathrm{j}}$ for all $\mathrm{j} \in\{1,2, \ldots,(\mathrm{N}-2)\}$ or $\mathrm{P}_{\mathrm{N}+1}=\left(\mathrm{P}_{\mathrm{N}}+\mathrm{A}_{1}\right)=2+\sum_{j=1}^{N} h j$ when $\mathrm{j} \epsilon\{1,2, \ldots, \mathrm{N}\}$. Here $\left(\mathrm{P}_{\mathrm{N}}+\mathrm{A}_{1}\right)=\mathrm{P}_{\mathrm{N}+1}$ or $\mathrm{P}_{\mathrm{N}-1}$, depends on the sign of $\mathrm{A}_{1}$.

$\mathrm{P}_{\mathrm{N}-1}=\mathrm{P}_{\mathrm{N}}+\mathrm{A}_{1}=2+\sum_{j=1}^{N-2} h j\left(\right.$ when $\left.\mathrm{A}_{1}<0\right)$. If $\mathrm{A}_{1}>0, \mathrm{P}_{\mathrm{N}+1}=\mathrm{P}_{\mathrm{N}}+\mathrm{A}_{1}=2+\sum_{j=1}^{N} h j$

Consider the case that $\mathrm{A}_{1}<0$.

Then $\left(\mathrm{A}_{1}-2\right)=-\mathrm{P}_{\mathrm{N}}+\sum_{j=1}^{N-2} h j$. Then $\left(2-\mathrm{A}_{1}\right)=\mathrm{P}_{\mathrm{N}}-\sum_{j=1}^{N-2} h j$

Then $\left(2-\mathrm{A}_{1}\right)=\mathrm{P}_{\mathrm{N}}-\mathrm{k}^{\prime}-\sum_{j=1}^{N-2} h j \ldots \ldots \ldots \ldots \ldots \ldots \ldots . .(21)$ Because here inside the term $\sum_{j=1}^{N-2} h j$, I have included (+ k') term.

But by $2^{\text {nd }}$ reference: for all $€>0$, there is a natural number ' $m$ ' such that for all $(\mathrm{N}-2)>m$; $\mathrm{h}_{\mathrm{N}-2}<\mathrm{P}_{\mathrm{N}-2} \cdot \mathrm{C}$

Let $\epsilon_{\mathrm{s}}$ is a positive real number $\epsilon_{\mathrm{s}}=\left[-\mathrm{B}+\mathrm{C}_{\mathrm{s}}+\mathrm{k}^{\prime}+\mathrm{P}_{\mathrm{N}}+\mathrm{B}_{2} . \mathrm{P}_{3}\right] / \mathrm{P}_{\mathrm{s}}>0$, such that $\mathrm{h}_{\mathrm{s}}<\mathrm{P}_{\mathrm{s}} * \epsilon_{\mathrm{s}}$ for all $s>(N-3)$. Let here the chosen $\epsilon_{s}$ implies that $m=(N-3)$ (Here $s$ is going from 1 to $(\mathrm{N}-2)$. Then " for all $\mathrm{s}>(\mathrm{N}-3)$ " means $\mathrm{s}=(\mathrm{N}-2)$. Where $\mathrm{k}$ ' is an integer number which not equals to 0 and we choose k' such that $\mathrm{k}^{\prime} /(\mathrm{N}-2)$ is an integer. Here the chosen k' integer number is responsible for $\mathrm{h}_{\mathrm{s}}<\mathrm{P}_{\mathrm{s}} * \epsilon_{\mathrm{s}}$ for all $\mathrm{s}>(\mathrm{N}-3)$ (i.e. $\left.\mathrm{s}=\mathrm{N}-2\right)$ and $\epsilon_{\mathrm{s}}>0$. That means here the value of $\mathrm{k}^{\prime}$ is responsible to say " $\epsilon_{\mathrm{s}}$ is existing such that $\mathrm{h}_{\mathrm{s}}<\mathrm{P}_{\mathrm{s}}{ }^{*} \epsilon_{\mathrm{s}}$, for $\mathrm{s}=(\mathrm{N}-2)$ ".

Here $h_{j}=b_{j}-\left[k^{\prime} /(N-2)\right]$ for all $j<(N-2)=s$. And where $\Sigma b_{j}=B$ for $j<(N-2)=s$. Then for some $\mathrm{C}_{\mathrm{s}}, \mathrm{h}_{\mathrm{s}}=\mathrm{P}_{\mathrm{s}}^{*} \epsilon_{\mathrm{s}}-\mathrm{C}_{\mathrm{s}}$; here $\mathrm{s} \equiv(\mathrm{N}-2)$. ${ }^{* * *}$ the meaning of ' $\mathrm{j}$ ' is the order number and $h_{j}$ is the prime gap between $P_{j+1}$ and $P_{j}$. Please refer the below content and the $2^{\text {nd }}$ reference. But here we chose $\mathrm{C}_{\mathrm{N}-2}$ such that $\mathrm{h}_{\mathrm{N}-2}=\mathrm{P}_{\mathrm{N}-2} * \mathrm{C}_{\mathrm{N}-2}-\mathrm{C}_{\mathrm{N}-2}$

But $\mathrm{h}_{\mathrm{N}-2}=\mathrm{P}_{\mathrm{N}-2} * \epsilon_{\mathrm{N}-2}-\mathrm{C}_{\mathrm{N}-2}=\left(-\mathrm{B}+\mathrm{k}^{\prime}+\mathrm{P}_{\mathrm{N}}+\mathrm{B}_{2} . \mathrm{P}_{3}\right)$. Where $\mathrm{k}^{\prime}$ is a natural number. Now let's use the $2^{\text {nd }}$ reference to proceed further. $B y(21)$ : 
$\left(\mathrm{A}_{1}-2\right)=-\mathrm{P}_{\mathrm{N}}+\sum_{j=1}^{N-2} h j=-\mathrm{P}_{\mathrm{N}}+\left(-\mathrm{B}+\mathrm{k}^{\prime}+\mathrm{P}_{\mathrm{N}}+\mathrm{B}_{2} \cdot \mathrm{P}_{3}\right)+\mathrm{B}-(\mathrm{N}-2) \cdot\left[\mathrm{k}^{\prime} /(\mathrm{N}-2)\right]=\mathrm{B}_{2} \cdot \mathrm{P}_{3}$

Thus by (22): $\left(\mathrm{A}_{1}-2\right)=\mathrm{P}_{3} \cdot \mathrm{B}_{2}$. Thus there exist consecutive prime numbers $\mathrm{P}_{\mathrm{N}}$ and $\left(\mathrm{P}_{\mathrm{N}}+\mathrm{A}_{1}\right)$ both greater than $\left(P_{n-1}+2\right)$ such that $\left(A_{1}-2\right)=B_{2} \cdot P_{3}$; for integer $B_{2}(\neq 0)$.

Similar to above, if $A_{1}>0$, we can proceed with the similar steps to prove that $\left(A_{1}-2\right)=B_{2} \cdot P_{3}$; for integer $\mathrm{B}_{2}(\neq 0)$ when $\mathrm{A}_{1}>0$.

\section{Proof 1}

Let's prove the existence of an integer $\left(\mathrm{P}_{N}-2\right)\left(>\mathrm{P}_{\mathrm{n}-1}+2\right)$ such that $\left(\mathrm{P}_{\mathrm{N}}-2\right)=\mathrm{P}_{3} \cdot \mathrm{x}_{3}$ such that $\mathrm{P}_{3}$ is divisible by $\mathrm{x}_{3}$. But $\mathrm{x}_{3}^{2}$ does not divide $\mathrm{P}_{3}$ as below. Where $\mathrm{P}_{3}=(\mathrm{a} / 2) \cdot \mathrm{x}_{3}$. $\left(\mathrm{x}_{3}-1\right)$ for some odd integer a. And where 4 does not divide $\left(\mathrm{x}_{3}-1\right)$.

By $2^{\text {nd }}$ reference: $\mathrm{P}_{\mathrm{N}}=2+\sum_{j=1}^{N-1} g j$, where $\mathrm{g}_{\mathrm{j}}=\mathrm{P}_{\mathrm{j}+1}-\mathrm{P}_{\mathrm{j}}$ for all $\mathrm{j} \epsilon\{1,2, \ldots \ldots, \mathrm{N}-1\}$

Then $\left(\mathrm{P}_{\mathrm{N}}-2\right)=\sum_{j=1}^{N-1} g j$

But by $2^{\text {nd }}$ reference: for all $€>0$, there is a natural number ' $\mathrm{m}_{0}$ ' such that for all $\mathrm{N}>\mathrm{m}_{0}$; $\mathrm{g}_{\mathrm{N}}<\mathrm{P}_{\mathrm{N}} . €$.

Let $\epsilon_{\mathrm{s}}$ is a positive real number $\epsilon_{\mathrm{s}}=\left[-\mathrm{A}+\mathrm{C}_{\mathrm{s}}+\mathrm{x}^{2}{ }_{3} \cdot \mathrm{k}_{1}\right] / \mathrm{P}_{\mathrm{s}}>0$, such that $\mathrm{h}_{\mathrm{s}}<\mathrm{P}_{\mathrm{s}}{ }^{*} \epsilon_{\mathrm{s}}$ for all $s>(N-2)$. Let here the chosen $\epsilon_{s}$ implies that $m_{0}=(N-2)$ (Here $s$ is going from 1 to $N-1$. Then " for all $\mathrm{s}>(\mathrm{N}-2)$ " means $\mathrm{s}=(\mathrm{N}-1))$. Where $\mathrm{k}_{1}$ is an integer number which is not divisible by $\mathrm{x}_{3}$. And let $\mathrm{k}_{1}=\mathrm{a} \cdot\left(\mathrm{x}_{3}-1\right) / 2$. Where ' $\mathrm{a}$ ' is an odd integer which is not divisible by $\mathrm{x}_{3}$. And 4 does

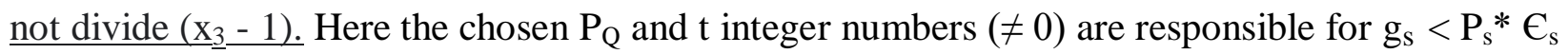
for all $\mathrm{s}>(\mathrm{N}-2)$ (i.e. $\mathrm{s}=\mathrm{N}-1)$ and $\mathrm{C}_{\mathrm{s}}>0$. OR in another words, the previously chosen integer $\mathrm{M}$ and $\mathrm{t}$ are responsible to say that above fact. That means here the value of $\mathrm{k}_{1}$ (or in another words, 'a') is responsible to say " $\epsilon_{\mathrm{s}}$ is existing such that $\mathrm{g}_{\mathrm{s}}<\mathrm{P}_{\mathrm{s}}{ }^{*} \mathrm{C}_{\mathrm{s}}$, for $\mathrm{s}=\mathrm{N}-1$ ". Here $\mathrm{g}_{\mathrm{j}}=$ $\mathrm{a}_{\mathrm{j}}$ for all $\mathrm{j}<(\mathrm{N}-1)=\mathrm{s}$. And where $\Sigma \mathrm{a}_{\mathrm{j}}=\mathrm{A}$ for $\mathrm{j}<(\mathrm{N}-1)=\mathrm{s}$. Then for some $\mathrm{C}_{\mathrm{s}}$, $\mathrm{g}_{\mathrm{s}}=\mathrm{P}_{\mathrm{s}} * \mathrm{C}_{\mathrm{s}}-\mathrm{C}_{\mathrm{s}}$; here $\mathrm{s} \equiv(\mathrm{N}-1)$. *** the meaning of ' $\mathrm{j}$ ' is the order number and $\mathrm{g}_{\mathrm{j}}$ is the prime gap between $P_{j+1}$ and $P_{j}$. Please refer the below content and the $2^{\text {nd }}$ reference. But here we chose $\mathrm{C}_{\mathrm{N}-1}$ such that $\mathrm{g}_{\mathrm{N}-1}=\mathrm{P}_{\mathrm{N}-1} * \mathrm{C}_{\mathrm{N}-1}-\mathrm{C}_{\mathrm{N}-1}$. 
But $\mathrm{g}_{\mathrm{N}-1}=\left(-\mathrm{A}+\mathrm{x}_{3}^{2} \cdot \mathrm{k}_{1}\right)$. Now let's use the $2^{\text {nd }}$ reference to proceed further.

By (23): $\left(\mathrm{P}_{\mathrm{N}}-2\right)=\sum_{j=1}^{N-1} g j$. But $\sum_{j=1}^{N-1} g j=\mathrm{A}+\left(-\mathrm{A}+\mathrm{x}_{3}^{2} \cdot \mathrm{k}_{1}\right)=\mathrm{x}_{3}^{2} \cdot \mathrm{k}_{1}$

Thus by (23) and (24): $\left(\mathrm{P}_{\mathrm{N}}-2\right)=\mathrm{x}_{3}^{2}$. $\mathrm{k}_{1}$; where $\mathrm{k}_{1}$ is not divisible by $\mathrm{x}_{3}$. Then $\left(\mathrm{P}_{\mathrm{N}}-2\right)=$ $\mathrm{x}_{3} \cdot\left(\mathrm{x}_{3} \cdot \mathrm{k}_{1}\right)=\mathrm{x}_{3} \cdot \mathrm{P}_{3} ;$ where $\mathrm{P}_{3}$ is divisible by $\mathrm{x}_{3}$.

Thus $\left(\mathrm{P}_{\mathrm{N}}-2\right)=\mathrm{P}_{3}$. $\mathrm{x}_{3}$; where $\mathrm{P}_{3}$ is divisible by $\mathrm{x}_{3}$. But $\mathrm{P}_{3}$ is not divisible by $\mathrm{x}_{3}^{2}$. Where 4 does not divide $\left(\mathrm{x}_{3}-1\right)$. Thus there exists an integer set $\left\{\mathrm{P}_{3} . \mathrm{x}_{3}\right.$ : where $\mathrm{P}_{3}$ is divisible by $\mathrm{x}_{3}$ but $\mathrm{P}_{3}$ is not divisible by $\mathrm{x}_{3}{ }_{3}$. Where 4 does not divide $\left(\mathrm{x}_{3}-1\right)$.

\section{Discussion}

We assumed initially that there are finitely many twin primes. After proceeding with that, I ended up with a contradiction. But to get the contradiction, I used that $\mathrm{P}_{\mathrm{N}}$ as a prime number greater than $\left(\mathrm{P}_{\mathrm{n}-1}+2\right)$. And we chose $\mathrm{P}_{\mathrm{n}}$ odd integer $(>1)$ and also we chose an integer $\mathrm{A}_{1}$ such that $\mathrm{P}_{3} \mid\left(\mathrm{A}_{1}-2\right)$. Also to get the contradiction, I used the facts that $\left(\mathrm{P}_{\mathrm{N}}+2\right)$ and $\left(\mathrm{P}_{\mathrm{N}}-2\right)$ as nonprimes since $\mathrm{P}_{\mathrm{N}}-2>\left(\mathrm{P}_{\mathrm{n}-1}+2\right)$. And also $\mathrm{I}$ have used that $\mathrm{x}_{2}$ and $\mathrm{x}_{3}$ as natural numbers (since, $\left(\mathrm{P}_{\mathrm{N}}+2\right)$ and $\left(\mathrm{P}_{\mathrm{N}}-2\right)$ are not prime numbers). And also $\mathrm{I}$ have used the fact (to get the contradiction as in (20) ): The difference between any two consecutive prime numbers (which are greater than $\left.\left(\mathrm{P}_{\mathrm{n}-1}+2\right)\right)$ is greater than 2. Therefore to get the contradiction, I have used the facts got from our assumption (1.0). Then the only possibility is our assumption (1.0) is false.

\section{Results}

Therefore I have used our assumption (1.0) to get the contradiction finally, as showed in (20). Therefore it is possible to conclude that our assumption (1.0) is false. Thus the negation of the assumption (1.0) is true.

Thus there are infinitely many twin prime numbers. 


\section{Appendix}

Prime number: A natural number which divides by 1 and itself only.

Twin Prime Numbers: Two prime numbers which have the difference exactly 2.

We denote ' $\mathrm{i}$ ' th prime gap $\mathrm{g}_{\mathrm{i}}=\mathrm{P}_{\mathrm{i}+1}-\mathrm{P}_{\mathrm{i}}$

Then according to the $2^{\text {nd }}$ reference; Prime number $\mathrm{P}_{\mathrm{N}}=2+\sum_{j=1}^{N-1} g j$

Also by $2^{\text {nd }}$ reference: for all $€>0$, there is a natural number ' $n$ ' such that for all $N-1>n$; $\mathrm{g}_{\mathrm{N}-1}<\mathrm{P}_{\mathrm{N}-1} \cdot \mathrm{C}$

\section{References}

1. Zhang, Yitang (2014). "Bounded gaps between primes". Annals of Mathematics. 179 (3): $1121-1174$.

2. https://ipfs.io/ipfs/QmXoypizjW3WknFiJnKLwHCnL72vedxjQkDDP1mXWo6uco/wiki/ Prime_gap.html

3. Terry Tao, Small and Large Gaps between the Primes

4. Maynard, James (2015), "Small gaps between primes", Annals of Mathematics, Second Series, 181 (1): 383-413

5. Tchudakoff, N. G. (1936). "On the difference between two neighboring prime numbers". Math. Sb. 1: 799-814.

6. Ingham, A. E. (1937). "On the difference between consecutive primes" Quarterly Journal of Mathematics. Oxford Series. 8 (1): 255-266. 